\title{
Prevalencia de parásitos gastrointestinales en vacas lecheras de sistema de producción tipo familiar
}

\section{Prevalence of gastrointestinal parasites in dairy cows of family-type production}

\author{
system
}

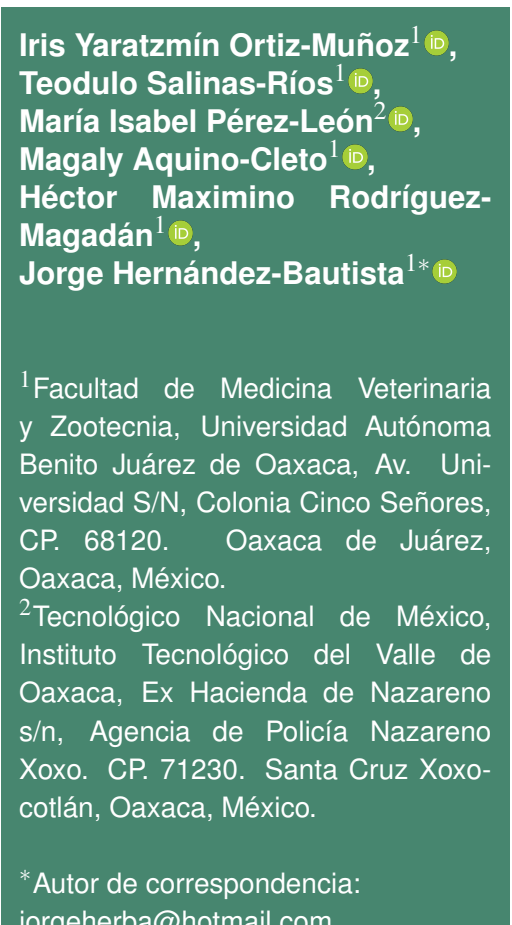

jorgeherba@hotmail.com

Artículo científico

Recibido: 27 de febrero 2021

Aceptado: 08 de octubre 2021

Como citar: Ortiz-Muñoz IY Salinas-Ríos T, Pérez-León MI, Aquino-Cleto M, Rodríguez-Magadán HM, Hernández-Bautista J (2021) Prevalencia de parásitos gastrointestinales en vacas lecheras de sistema de producción tipo familiar. Ecosistemas y Recursos Agropecuarios Núm. Esp. II: e2920. DOI: 10.19136/era.a8nll.2920
RESUMEN. La presencia de endoparásitos es un problema frecuente que afecta la salud y productividad de hatos lecheros. El objetivo fue determinar la relación entre la condición corporal, número de partos y época del año con la prevalencia de parásitos gastrointestinales en vacas de lechería familiar en los distritos de Etla y Zimatlán de Álvarez, Oaxaca, México. El estudio se realizó en primavera, verano, otoño e invierno, con 185 vacas Holstein (116 de Etla y 69 de Zimatlán de Álvarez), con diferente condición corporal $(\leq 2.0,2.5$ y $\geq 3$ ) y número de partos (1-3 y 4-6). Las muestras fecales se analizaron mediante la técnica de flotación con solución saturada de cloruro de sodio. Los datos se sometieron a un análisis de varianza a través de un modelo de datos categóricos $(p<0.05)$, considerando como efectos fijos la condición corporal, número de partos, época del año y distritos. El $66 \%$ de las vacas presentaron huevos y ooquistes de parásitos intestinales, no habiendo diferencia entre distritos. De los parásitos analizados, Eimeria spp. fue el de mayor prevalencia en ambos distritos. El porcentaje de vacas con Cryptosporidium, Strongyloides y Oesophagostomum fue mayor $(p<0.05)$ en Zimatlán de Álvarez. El porcentaje de vacas con parásitos gastrointestinales se incrementaron $(p<0.05)$ en primavera y verano. Se concluye que las condiciones climáticas, la condición corporal y el número de partos son factores de riesgo para la presentación de parásitos gastrointestinales, principalmente de Eimeria spp. en vacas de lechería familiar de los distritos de Etla y Zimatlán de Álvarez.

Palabras clave: Condición corporal, Eimeria spp., época del año, Holstein, parasitosis.

ABSTRACT. Endoparasites is a common problem affecting mainly dairy herds health and productivity. The objective was to determine the relationship between body condition, number of births and time of year with the prevalence of gastrointestinal parasites in family dairy cows established in District of Etla and Zimatlán de Álvarez, Oaxaca, México. The study was carried out in spring, summer, autumn and winter, using 185 Holstein cows (116 from Etla and 69 from Zimatlán de Alvarez), with different body condition $(\leq 2.0,2.5$ and $\geq 3$ ) and number of births (1-3 and 4-6). Fecal samples were analyzed using flotation technique with saturated sodium chloride solution. Data were analyzed with a variance analysis through a categorical data model ( $p<0.05$ ), considering as fixed effects body condition, number of births, time of year and districts. $66 \%$ of cows had endoparasites, with no difference between districts. Of the parasites analyzed, Eimeria spp. it was the most prevalence in both districts. The percentage of cows with Cryptosporidium, Strongyloides and Oesophagostomum were higher $(p<0.05)$ in Zimatlán de Álvarez. The number of cases increased $(p<0.05)$ in spring and summer. It is concluded that climatic conditions, body condition and number of births contribute to increased gastrointestinal parasites, mainly Eimeria spp., in family dairy cows in the Districts of Etla and Zimatlán of Alvarez.

Key words: Corporal condition, Eimeria spp., time of year, Holstein, parasitism. 


\section{INTRODUCCIÓN}

La producción y venta de leche representan un ingreso constante para los ganaderos productores de leche. En Valles Centrales de Oaxaca, principalmente en los distritos de Etla y Zimatlán de Álvarez se elabora el denominado quesillo (queso tipo Oaxaca). Aunque en Zimatlán de Álvarez se produce 11.5 veces más leche que en Etla (SIAP 2019), en ambos distritos, el sistema de producción es de tipo familiar o de traspatio con pequeñas extensiones de terrenos (Sánchez-Vásquez et al. 2017, CamachoVera et al. 2020). La mayor parte de la producción de leche se vende a pie de establo a productores de queso, también hay productores que elaboran queso y lo venden directamente al consumidor (Martínez et al. 2012). La expansión de la lechería se ve limitada por la disponibilidad de terreno cultivable y la carencia constante de agua debido a la reducción del manto freático (Sánchez-Medina et al. 2017). El manejo sanitario realizado al ganado lechero es curativo, sin un calendario preventivo que incluya análisis clínicos y que permita preservar la salud de éstos (Hine et al. 2012).

La presencia de parásitos en el sistema digestivo es un problema frecuente que afecta la salud y productividad de la vaca; además, la resistencia a desparasitantes, contaminación de suelo y agua, mal manejo sanitario y baja respuesta inmunológica del rumiante, además la pobre condición corporal dificulta su control (Sordillo 2016), ocasionando procesos infecciosos, o manteniéndose de manera subclínica (Colina et al. 2013). Los animales infestados pueden llegar a disminuir de 10 a $25 \%$ la producción y fertilidad, aunque en apariencia pueden mostrar buen estado de salud (Huang et al. 2014, Bellet et al. 2018).

La presencia de endoparásitos en el hato, como coccidiosis y helmintos (Fasciola, Equinococcus, Spirometra, Ascaris, Trichuris, Toxacara, Ancylostoma, Strongyloides, entre otros), implica un riesgo sanitario significativo, ya que, algunos de estos organismos son transmitidos a otras especies y al humano provocando alteraciones en la salud, por ejemplo, E. granulosus y E. multilocu- laris que causan problemas gastrointestinales, Ascaris suum y Trichuris trichiura retraso cognitivo y retraso en el crecimiento (Robinson y Dalton 2009, Karshima et al. 2018). Al respecto, Rodríguez-Vivas et al. (2017) mencionan que, en México las pérdidas económicas provocadas por Eimeria spp. fue de 23.78 millones de dólares en el año 2013, debido a que las condiciones ambientales del país son propicias para la proliferación de estos parásitos. Además, en regiones del país con climas tropicales y subtropicales, se ha registrado presencia de nematodos de las especies Haemonchus placei, $H$. similis, $H$. contortus, Mecistocirrus digitatus, Cooperia punctata, C. pectinata, Trichostrongylus axei y Oesophagostomum radiatum (Vázquez et al. 2004).

El diagnóstico de parásitos gastrointestinales, mediante la búsqueda de larvas y huevos en las heces (Pinilla et al. 2018), no solo es útil para animales enfermos, sino para aquellos con riesgo metabólico, reproductivo e infeccioso; así como para la toma de decisiones preventivas y evitar pérdidas económicas (Charlier et al. 2016). Sumado a esto, la descripción e identificación de factores de riesgo que limitan el rendimiento animal podría considerarse un componente de la enfermedad; por lo que, redefinir estos procesos de forma más amplia para incluir afecciones subclínicas, es igualmente importante para el paradigma de gestión de la salud (LeBlanc et al. 2006). Por lo anterior, el objetivo del estudio fue determinar la relación entre la condición corporal, número de partos y época del año con la prevalencia de parásitos gastrointestinales en vacas de lechería familiar establecidas en los distritos de Etla y Zimatlán de Álvarez, Oaxaca, México.

\section{MATERIALES Y MÉTODOS}

\section{Área de estudio}

El estudio se realizó en los distritos de Etla y Zimatlán de Álvarez, en la región de los Valles Centrales del estado de Oaxaca. El distrito de Etla se localiza en las coordenadas $17^{\circ}, 12^{\prime} \mathrm{LN}$ y $96^{\circ}$, 48' LO, a $1660 \mathrm{msnm}$; tiene una extensión territorial de $2220.96 \mathrm{~km}^{2}$, temperatura promedio de 22 ${ }^{\circ} \mathrm{C}$ y precipitación pluvial de $730.6 \mathrm{~mm}$. El distrito 
de Zimatlán de Álvarez se localiza entre las coordenadas $6^{\circ} 52^{\prime} 00^{\prime \prime}$ LN y $96^{\circ} 46^{\prime} 60$ " LO, a $1499 \mathrm{msnm}$, posee una extensión territorial de $988.49 \mathrm{~km}^{2}$, temperatura promedio de $20.9{ }^{\circ} \mathrm{C}$ y precipitación de 709 mm (INAFED 2017).

\section{Muestreo}

Se realizó un muestreo no probabilístico denominado bola de nieve, en donde los propietarios de las primeras unidades de producción intervenidas recomendaron a los demás propietarios; debido a que se desconocía la ubicación de las unidades de producción. En total se estudiaron 116 vacas en el distrito de Etla y 69 vacas en el distrito de Zimatlán de Álvarez. En ambos distritos la dieta se basó en alfalfa fresca y rastrojo de maíz, además de ofrecer sal mineral y agua a libre acceso. La recolección de muestras de heces se realizó al momento de la primera ordeña (5:00 am), en cada época del año del 2017 (en primavera del 10 al 29 de abril, en verano del 1 al 15 de agosto, en otoño del 1 al 15 de octubre y en invierno del 20 al 30 de diciembre). Las heces se obtuvieron vía rectal, para luego colocarlas en vasos estériles de $200 \mathrm{~mL}$. Las muestras se mantuvieron a temperatura ambiente durante $20 \mathrm{~min}$ y posteriormente se refrigeraron a $4{ }^{\circ} \mathrm{C}$, hasta su procesamiento a las $24 \mathrm{~h}$ posteriores al muestreo.

\section{Variables analizadas}

Durante el muestreo se registraron las variables: condición corporal (tres niveles: $\leq 2,2.5$ y $\geq 3$; en la escala de 1 a 5 de acuerdo con Edmonson et al. (1989); la época del año (primavera, verano, otoño e invierno) y el número de partos (dos grupos: de 1 a 3 y de 4 a 6 ). En el estudio coprológico se utilizó la técnica de flotación en solución saturada con $\mathrm{NaCl}$ en frotis fecal de acuerdo con el protocolo descrito por Sandoval et al. (2011). Brevemente, se homogenizaron $200 \mathrm{~mL}$ de solución con $10 \mathrm{~g}$ de heces, filtrada la mezcla se recolectaron gotas de la superficie donde flotan huevos y ooquistes, colocándose en portaobjetos e inmediatamente después se adicionó Lugol para mejor contraste y observar en un microscopio óptico (Carl Zeiss ${ }^{M R}$, modelo K7). La diferenciación de huevos de nemátodos y ooquistes de protozoarios de los parásitos gastrointestinales se realizó por morfología (Zajac y Conboy 2011).

\section{Análisis estadístico}

Los datos obtenidos de prevalencia se sometieron a análisis de varianza ajustado a un modelo de datos categóricos con tres efectos fijos (condición corporal, época del año y número de partos) y tres interacciones dobles, considerando significancia estadística a un $\alpha=0.05$. Para determinar la proporción de casos se desarrolló un análisis de frecuencias.

\section{RESULTADOS}

El análisis estadístico demostró que las interacciones dobles entre los factores, condición corporal, número de partos y época del año, no resultaron significativas $(p>0.05)$; por lo que solo se presentan resultados de los efectos principales.

\section{Presencia de endoparásitos}

Se encontró que la proporción de casos positivos a parásitos gastrointestinales de Eimeria spp. y Ostertagia spp fueron similares $(p>0.05)$ en ambos distritos; por lo tanto, se reportan los promedios generales para cada especie, que fueron de 49.21 y $0.44 \%$, respectivamente. Mientras que el porcentaje de vacas con presencia de Strongyloides spp, Oesophagostomum spp. y del protozoario Cryptosporidium spp. fue mayor $(p \leq 0.05)$ en el distrito de Zimatlán de Álvarez (Tabla 1).

Tabla 1. Porcentaje de vacas positivas a parásitos gastrointestinales en el sistema de producción familiar establecido en los distritos de Etla y Zimatlán de Álvarez, Oaxaca, México.

\begin{tabular}{lcc}
\hline Tipo de parásito & $\begin{array}{c}\text { Distrito } \\
\text { de Etla }\end{array}$ & $\begin{array}{c}\text { Distrito de } \\
\text { Zimatlán }\end{array}$ \\
\hline Casos positivos (\%) & 62.28 & 72.46 \\
Eimeria spp. (\%) & 48.25 & 50.17 \\
Cryptosporidium (\%) & $7.89^{a}$ & $36.23^{b}$ \\
Strongyloides spp. (\%) & $24.56^{a}$ & $55.07^{b}$ \\
Ostertagia spp. (\%) & 0.88 & 0 \\
Oesophagostomum spp. (\%) & $3.51^{a}$ & $26.09^{b}$ \\
\hline${ }^{b}$ Letras distintas entre columnas indican diferencia es- \\
tadística (p $\leq$ 0.05).
\end{tabular}




\section{Efecto de la época del año}

En primavera se observó el mayor $(p \leq 0.05)$ porcentaje de casos de vacas con parásitos gastrointestinales; mientras que, en las otras tres épocas, el porcentaje de casos fue similar $(p>0.05)$, en promedio de $66 \%$ de vacas con presencia de parásitos (Tabla 2). En lo que se refiere a Eimeria spp., el menor $(p \leq 0.05)$ porcentaje de casos $(43.64 \%)$ se registró en invierno; mientras que en las épocas de primavera, verano y otoño se observaron valores similares $(p>0.05)$. El mayor porcentaje de casos de infección con Strongyloides spp. y Oesophagostomun spp. se detectó en primavera y verano, mientras que en invierno disminuyó, no detectándose casos en otoño ( $\mathrm{p} \leq 0.05$ ). Respecto a Cryposporidium spp., se observaron efectos similares a lo observado con los otros géneros, pero hubo presencia en todas las épocas, incrementando los casos $(p \leq 0.05)$ en las estaciones de primavera y verano.

\section{Efecto de la condición corporal}

De forma general, se detectó que el porcentaje de vacas con problemas de parásitos no fue afectado $(p>0.05)$ por la $C C$, pero determinó el tipo de parásito existente. La presencia de Eimeria spp. y Cryptosporidium spp. fue menor en vacas con CC menor o igual a $2(p \leq 0.05)$ comparadas con las de 2.5 y las de condición corporal mayor o igual a 3. Mientras que las vacas con CC de 2.5 fueron las únicas con Ostertagia spp. $(\mathrm{p} \leq 0.05)$ (Tabla 3$)$.

\section{Efecto del número de partos}

En vacas igual o mayor a siete partos fue donde se presentó el menor porcentaje de casos positivos a parásitos $(p \leq 0.05)$. Con respecto al tipo de parásito se observó que Strongyloides, Cryptosporidium, Ostertagia y Oesophagostomum son los que se detectaron con menor frecuencia en este grupo de vacas. Cabe resaltar que, en Eimeria spp. el comportamiento es inverso y significativo ( $p \leq 0.05)$, siendo las vacas jóvenes quienes presentan menor porcentaje de casos (Tabla 4).

\section{DISCUSIÓN}

\section{Presencia de parásitos gastrointestinales}

En promedio se encontró $66 \%$ de vacas con parásitos gastrointestinales. Datos similares a lo reportado por Marskole et al. (2016), quienes reportaron en granjas de Jabalpur una prevalencia del $75 \%$ de parásitos gastrointestinales en vacas. Además, ambos distritos mostraron presencia de Eimeria spp. y Ostertagia spp. con promedios generales similares. Mientras que en Zimatlán de Álvarez se tuvo el mayor porcentaje de vacas con Strongyloides spp., Oesophagostomum spp y Cryptosporidium spp. Se conoce que los géneros de parásitos gastrointestinales Ostertagia spp., Oesophagostomum spp. y Strongyloides spp. por su incidencia y patogenicidad puede ocasionar signos graves en el tubo digestivo de los bovinos (Steffan et al. 2012). Dichos géneros son de ciclo directo y ocasionan una infección múltiple, la cual se identificó en las vacas de ambos distritos; al respecto Orantes-Zebuada et al. (2014) reportan una incidencia del $7.3 \%$ de huevos de parásitos en 22 municipios de la región centro de Chiapas. En contraste, en un estudio realizado en Aguascalientes y la región de los Altos de Jalisco (Cruz-Vázquez et al. 2017) se observó una prevalencia de protozoarios del 59\%. El porcentaje de casos de parásitos encontrados en el presente estudio, son similares a los obtenidos en las vacas establecidas en los estados de Aguascalientes y Jalisco; pero se encuentra muy por encima del porcentaje de casos reportados para el estado de Chiapas, lo que se puede deber a que, aunque Oaxaca y Chiapas tienen el mismo sistema de traspatio y condiciones climáticas iguales, en Oaxaca podría haber más contaminación de parásitos en el suelo y agua utilizados para la alimentación del ganado.

\section{Parasitosis respecto a época del año}

Durante la primavera la prevalencia de parásitos gastrointestinales fue superior con respecto a las demás estaciones del año. De manera interesante, Eimeria spp. se presentó en menor porcentaje durante el invierno, pero con el mismo porcentaje entre las otras tres épocas. Esto se puede deber a 
Tabla 2. Porcentaje de vacas positivas a parásitos gastrointestinales, en cuatro épocas del año, en el sistema de producción familiar establecido en los distritos de Etla y Zimatlán de Álvarez, Oaxaca, México.

\begin{tabular}{lcccc}
\hline Tipo de parásito & Primavera & Verano & Otoño & Invierno \\
\hline Casos positivos (\%) & $75.61^{b}$ & $64.29^{a}$ & $61.29^{a}$ & $63.64^{a}$ \\
Eimeria spp. (\%) & $51.22^{b}$ & $50.00^{b}$ & $54.84^{b}$ & $43.64^{a}$ \\
Cryptosporidium (\%) & $56.10^{c}$ & $46.43^{b c}$ & $35.48^{b}$ & $10.91^{a}$ \\
Strongyloides spp. (\%) & $26.83^{c}$ & $26.79^{c}$ & $0^{a}$ & $14.55^{b}$ \\
Ostertagia spp. (\%) & 0 & 0 & 0 & 1.82 \\
Oesophagostomum spp. (\%) & $19.51^{b}$ & $17.86^{b}$ & $0^{a}$ & $7.27^{a}$ \\
\hline \multicolumn{7}{l}{ abc Letras distintas entre columnas indican diferencia estadística $(\mathrm{p} \leq 0.05)}$.
\end{tabular}

Tabla 3. Porcentaje de vacas de diferente condición corporal positivas a parásitos gastrointestinales en el sistema de producción familiar establecido en los distritos de Etla y Zimatlán de Álvarez, Oaxaca, México.

\begin{tabular}{lccc}
\hline \multirow{2}{*}{ Tipo de parásito } & \multicolumn{3}{c}{ Condición corporal } \\
\cline { 2 - 4 } & Menor o igual a 2 & 2.5 & Mayor o igual a 3 \\
\hline Casos positivos (\%) & 60.87 & 67.29 & 70.00 \\
Eimeria spp. (\%) & $39.13^{a}$ & $50.97^{b}$ & $60.00^{b}$ \\
Cryptosporidium (\%) & $26.09^{a}$ & $40.19^{b}$ & $36.67^{b}$ \\
Strongyloides spp. (\%) & $15.22^{a}$ & $22.93^{b}$ & $10.00^{a}$ \\
Ostertagia spp. (\%) & 0 & 0.93 & 0 \\
Oesophagostomum spp. (\%) & $6.52^{a}$ & $17.76^{b}$ & $0^{a}$ \\
\hline${ }^{a}$ Letras distintas entre columnas indican diferencia estadística $(\mathrm{p}<0.05)$
\end{tabular}

que este protozoario se reproduce óptimamente a temperaturas entre 20 y $34^{\circ} \mathrm{C}$ (Barreto et al. 2015). Además, estos resultados son similares a los reportados para el ganado de Yucatán, donde se observó presencia de tres especies de Eimeria a lo largo de todo el año, debido a que la temperatura anual se encuentra entre 24.1 y $29.4^{\circ} \mathrm{C}$ (Vázquez et al. 2004).

Tabla 4. Porcentaje de vacas, de diferente número de partos, positivas a parásitos intestinales, en el sistema de producción familiar establecido en los distritos de Etla y Zimatlán, Oaxaca, México.

\begin{tabular}{lccc}
\hline \multirow{2}{*}{ Tipo de parásito } & \multicolumn{3}{c}{ Número de partos } \\
\cline { 2 - 4 } & $\leq 3$ & Entre 4 y 6 & $\geq 7$ \\
\hline Casos positivos (\%) & $69.23^{b}$ & $67.84^{b}$ & $44.44^{a}$ \\
Eimeria spp. (\%) & $42.31^{a}$ & $55.17^{b}$ & $50.00^{b}$ \\
Cryptosporidium (\%) & $39.74^{b}$ & $34.48^{a}$ & $27.78^{a}$ \\
Strongyloides spp. (\%) & $23.08^{c}$ & $17.24^{b}$ & $5.56^{a}$ \\
Ostertagia spp. (\%) & 1.28 & 0 & 0 \\
Oesophagostomum spp. (\%) & $15.38^{b}$ & $11.49^{b}$ & $0^{a}$ \\
\hline$a b c$ Letras distintas en columnas indican diferencia estadística (p $\leq 0.05)$.
\end{tabular}

El mayor porcentaje de casos de infección con los géneros de Strongyloides spp. y Oesophagostomun spp. se observaron en primavera y verano, mientras que en invierno y otoño disminuyeron. Con respecto a los casos de Cryptosporidium spp., en todas las épocas se encontró, pero incrementaron en primavera y verano. Estudios realizados en diferentes épocas del año en los estados de Yucatán, Nayarit y Veracruz han observado alta incidencia de parásitos con diferencia en la presentación, siendo mayor en verano y disminuyendo en invierno (Vázquez et al. 2004, Anzures-Olvera et al. 2015)

\section{Parasitosis en relación a la condición corporal}

La condición corporal (CC) per se, puede considerarse como un reflejo de salud en vacas (CariziCherobin et al. 2019), en el cual, se considera que vacas lecheras con una $\mathrm{CC}$ de 2.5 a 3 como adecuada "delgada saludable" y una CC menor a esta como animales más propensos a presentar alteraciones metabólicas e infecciones (Roche et al. 2009, Bell et al. 2018). En este estudio las vacas con la CC más baja a pesar de no ser estadísticamente significativa $(p>0.05)$ fueron las que presentaron menor número de casos positivos, por lo que se puede considerar que son resistentes a infecciones parasitarias. La CC mayor de 3 presentó mayor número de casos positivos, por lo que estas vacas pueden ser consideradas como resilientes (con producción aceptable, con habilidad para mantenerse sanas a pesar de la infección parasitaria) lo cual es 
compatible con los resultados obtenidos por Morales et al. (2012). El control de la carga parasitaria es importante ya que se ha demostrado que vacas con parásitos producen menos leche que aquellas con carga nula (Perri et al. 2011).

\section{Parasitosis en relación al número de partos}

El número de partos se puede relacionar con la edad de las vacas (Mariscal-Aguayo et al. 2016). En este trabajo se encontró que vacas con el mayor número de partos presentaron mayor prevalencia de Strongyloides, Cryptosporidium, Ostertagia y Oesophagostomum. Al respecto, Gunathilaka et al. (2018) encontraron que vacas mayores de cinco años tienen menor tasa de infección por parásitos que las más jóvenes. Esto se puede deber a la mayor exposición a parásitos y en consecuencia respuesta inmune más eficiente (Hendawy 2018, Rodríguez-Vivas et al. 2017). Mientras que, para la presencia de Eimeria spp. el comportamiento fue inverso, ya que las vacas jóvenes mostraron menor porcentaje de casos; contrario a lo reportado por Huang et al. (2014) quienes mencionan que vacas mayores a 2 años presentan menor cantidad (5.1\%) de Eimeria spp. en comparación con vacas de 1 a 2 años (16.9\%) y de menores de 1 año (14.8\%). Nuestros datos también se contraponen a lo encontrado en vacas lecheras del trópico alto de Antioquia, Colombia, donde becerros menores de 1 año mostraron mayor presencia $(70.5 \%)$ que vacas de más de 1 año (40\%). Al respecto, Arellano et al. (2006) mencionan que las vacas con genotipo Holstein con 3 a 4 partos aún se encuentran en periodo de crecimiento por lo que pueden tener mayor susceptibilidad a esta parasitosis.

A pesar de presentar coincidencias en cuanto a sistemas de producción, alimentación y manejo, las vacas de Etla físicamente presentaron condiciones no óptimas en su CC que se reflejan en menor producción de leche en relación con las vacas de Zimatlán de Álvarez, que, a pesar de tener mejor condición corporal la presencia de parásitos se mantiene. Al respecto, se sabe que el factor ambiental tiene efecto en la presencia de parásitos, por ejemplo, la distribución de la precipitación y las características del suelo. Estas variaciones son determinantes en la viabilidad y maduración de larvas y huevos de geohelmintos patógenos, así como de protozoarios, siendo estos los más resistentes a tratamientos de agua como es el uso de oxidantes como el cloro (Silva et al. 2005, OMS 2017). Específicamente en Zimatlán de Álvarez se ha reportado que las aguas han sido afectadas por la contaminación municipal e industrial del Río Salado y el Río Atoyac (SorianoHernández et al. 2017). Por lo que profundizar en la identificación de las fuentes de infección ayudaría a comprender la distribución parasitaria desde un punto de vista epidemiológico, así como el planteamiento de estrategias que lleven a un control adecuado.

\section{CONCLUSIONES}

Dos terceras partes de las vacas en las unidades de producción de lechería familiar de los distritos de Etla y Zimatlán de Álvarez fueron positivas por lo menos un parásito gastrointestinal; y la mitad de las vacas de ambos distritos poseen carga parasitaria de Eimeria spp. La presencia de parásitos gastrointestinales en vacas lecheras aumenta en la época de primavera, sobre todo en vacas con menor número de partos, pero con la mayor condición corporal, por lo que es recomendable darle un mejor manejo sanitario durante estas condiciones.

\section{LITERATURA CITADA}

Anzures-Olvera F, Macías-Cruz U, Álvarez-Valenzuela FD, Correa-Calderón A, Díaz-Molina R, Hernández-Rivera JA, Avendaño-Reyes L (2015) Efecto de época del año (verano vs invierno) en variables fisiológicas, producción de leche y capacidad antioxidante de vacas Holstein en una zona árida del noreste de México. Archivo de Medicina Veterinaria 47: 15-20.

Arellano S, Martínez J, Romero E, Briones F, Domínguez M, De la Garza F (2006) Factores genéticos-ambientales 
que afectan el intervalo entre partos y días a primer parto en ganado de doble propósito en el norte de Veracruz. Avances en Investigación Agropecuaria 10: 43-53.

Barreto SLE, Ferreira CJ, Texeira NMR, Rego AG, Brandao MAD, Tapia TMD (2015) Epidemiology or Eimeria infections in sheep raised extensively in a semiarid region of Brazil. Brazil Journal of Veterinary Parasitology 24: 410-415.

Bell MJ, Maak M, Sorley M, Proud R (2018) Comparison of methods for monitoring the body condition of dairy cows. Frontiers in Sustainable Food System 26: 2. Article 80. DOI: 10.3389/fsufs.2018.00080.

Bellet C, Green MJ, Bradley AJ, Kaler J (2018) A longitudinal study of gastrointestinal parasites in English dairy farms. Practices and factors associated with first lactation heifer exposure to Ostertagia ostertagi on pasture. Journal of Dairy Science 101: 537-546.

Camacho-Vera JH, Cervantes-Escoto F, Palacios-Rangel MA (2020) Historia y raíces artesanales de un sistema tradicional: la producción de queso en Reyes Etla, Oaxaca. Revista de Alimentación Contemporánea y Desarrollo Regional 30: 1-29.

Carizi-Cherobi V, Garzón PJP, Alvarado MJP, Marini PR (2019) Condición corporal y su relación con producción láctea, reproducción y perfil metabólico en vacas lecheras del trópico boliviano. Revista de Investigación Veterinaria de Perú 30: 107-118.

Charlier J, De Waele V, Ducheyne E, van der Voort M, Velde FV, Claerebout E (2016) Decision making on helminths in cattle: diagnostics, economics and human behaviour. Irish Veterinary Journal 69: 14 DOI: 10.1186/s13620-016-0073-6.

Colina CJ, Mendoza CG, Jara AC (2013) Prevalencia e Intensidad del parasitismo gastrointestinal por nemátodos en bovinos, Bos taurus, del distrito Pacanga (La Libertad, Perú). Revista Científica de la Facultad de Ciencias Biológicas de Nacional de Trujillo 33: 76-83.

Cruz-Vásquez C, Vital-Gutiérrez J, Medina-Esperanza L, Ortega-Mora L, Valdivia-Flores A, Quesada-Tristán T, Orihuela-Trujillo A (2017) Neospora caninum infection during the first gestation of Holstein Heifers that consume food contaminated naturally with Zearalelone under field conditions. Iran journal of Parasitology 12: $563-571$.

Edmonson AJ, Lean IJ, Weaver LD, Farver T, Webster G (1989) A body condition scoring chart for Holstein dairy cows. Journal of Dairy Science 72: 68-78.

Gunathilaka N, Niroshana D, Amarasinghe D, Udayanga L (2018) Prevalence of Gastrointestinal Parasitic Infections and Assessment of Deworming Program among Cattle and Buffaloes in Gampaha District, Sri Lanka. BioMed Research International 2018: Article ID 3048373. DOI: 10.1155/2018/3048373.

Hendawy HMS (2018) Immunity to gastrointesnital nematodes in ruminants: effector cell mechanism and cytokines. Journal of Parasites Disease 42: 471-482.

Hine BC, Shannon L. Cartwright, Bonnie A, Mallard (2012) Analysis of leukocyte populations in Canadian Holsteins classified as high or low immune responders for antibody- or cell-mediated immune response. Canadian Journal of Veterinary Research 76: 149-156.

Huang CC, Lian-Chen W, Chien-Hung P, Cheng-Hsiung Y, Chen-Hung L (2014) Investigation of gastrointestinal parasites of dairy cattle around Taiwan. Journal of Microbiology, Immunology and Infection 47: 70-74.

INAFED (2017) Enciclopedia de los Municipios y Delegaciones de México: Estado de Oaxaca. http://www.inafed. gob.mx/work/enciclopedia/EMM20oaxaca/index.html. Fecha de consulta: 12 de enero de 2020. 
Karshima SN, Maikai BV, Kwaga JKP (2018) Helmints of veterinary and zoonotic importance in Nigeria Ruminants: a 46-year meta-analysis (1970-2016) of their prevalence and distribution. Infectious diseases of poverty 7(1): 52. DOI: 10.1186/s40249-018-0438-z.

LeBlanc SJ, Lissemore KD, Kelton DF, Duffield TF, Leslie KE (2006) Major advances in disease prevention in dairy cattle. Journal of Dairy Science 89: 1267-79.

Mariscal-Aguayo V, Pacheco-Cervantes A, Estrella-Quintero E, Huerta-Bravo M, Rangel-Santos R, Núñez-Domínguez R (2016). Indicadores reproductivos de vacas lecheras en agroempresas con diferente nivel tecnológico en los Altos de Jalisco. Agricultura, Sociedad y Desarrollo 13: 493-507.

Marskole P, Verma Y, Dixit AK, Swamy M (2016) Prevalence and burden of gastrointestinal parasites in cattle and buffaloes in Jabalpur, India. Veterinary World 9: 1214-1217.

Martínez CCJ, Cotera RJ, Abad ZJ (2012) Características de la producción y comercialización de leche bovina en sistemas de doble propósito en Dobladero, Veracruz. Revista Mexicana de Agronegocios 30: 816-824.

Morales G, Arelis P L, Sandoval E, Jiménez D, Morales J (2012) Relationship between body condition and level of parasite infestation in grazing cattle as a criterion for selective deworming. Revista de Investigaciones Veterinarias del Perú 23: 80-89.

Orantes-Zebadua MA, Platas-Rosado D, Cordova-Avalos V, De los Santos-Lara MA, Córdova-Avalos A (2014) Caracterización de la ganadería de doble propósito en una región de Chiapas, México. Ecosistemas y Recursos Agropecuarios 1: 49-58.

OMS (2017) Helmintiasis transmitida por el suelo, Nota descriptiva, Organización Mundial de la Salud https://www. who.int/es/news-room/fact-sheets/detail/soil-transmitted-helminth-infections. Fecha de consulta: 2 de marzo de 2020.

Perri A F, Mejía M E, Licoff N, Lazaro L, Miglierina M, Ornstein A, Becu-Villalobos D, Lacau-Mendigo IM (2011) Gastrointestinal parasites presence during the peripartum decreases total milk production in grazing dairy Holstein cows. Veterinary parasitology 178: 311-318.

Pinilla JC, Flórez P, Sierra M, Morales E, Sierra R, Vásquez MC, Tobon JC, Sánche A, Ortíz D (2018) Prevalencia del parasitismo gastrointestinal en bovinos del departamento Cesar, Colombia. Revista Internacional Veterinaria Perú 29: 278-287.

Robinson MW, Dalton JP (2009) Zoonotic helminth infections with particular emphasis on fasciolosis and other trematodiasis. Philosophical Transactions of the Royal Society B 364: 2763-2776.

Roche JR, Friggens NC, Kay JK, Fisher MW, Stafford KJ, Berry DP (2009) Invited review: Body condition score and its association with dairy cow productivity, health, and welfare. Journal of Dairy Science 92: 5769-5801.

Rodríguez-Vivas RI, Grisi L, Pérez-de León AA, Silva-Villela H, Torres-Acosta JFJ, Fragoso HS, Romero DS, Rosario RC, Saldierna F, García DC (2017) Potential economic impact assessment for cattle parasites in Mexico. Review. Revista Mexicana de Ciencias Pecuarias 8: 61-74.

Sánchez-Medina AP, Herrera-Haro JG, Ramírez-Bribiesca JE, Ortega-Cerrilla ME, Mendoza-Nazar P, DorantesJimenez J (2017) Evaluación económica del sistema de producción lechera familiar. Agroproductividad 11: 111-117.

Sánchez-Vásquez V, Ruí-Martínez A, Duran-Meléndez E, Gijón-Cruz AS, Maciel-Noyola MC (2017) La cadena productiva de bovinos productores de leche bajo el enfoque competitivo del cluster en la región de los Valles Centrales de Oaxaca, México. Revista Mexicana de Agronegocios 41: 708-719. 
Sandoval E, Morales G, Ybarra N, Barrios M, Borges J (2011) Comparación entre dos modelos diferentes de cámaras de McMaster empleadas para el conteo coproscópico en el diagnóstico de infecciones por nematodos gastroentéricos en rumiantes. Zootecnia Tropical 29: 495-501.

SIAP (2019) Anuario estadístico de la producción ganadera. Servicio de Información Agroalimentaria y Pesquera https://nube.siap.gob.mx/cierre_pecuario/. Fecha de consulta: 3 de febrero de 2021.

Silva J, Torres P, Madera C (2008) Reusos de aguas residuales domésticas en agricultura. Una revisión. Agronomía Colombiana 26: 347-359.

Sordillo LM (2016) Nutritional strategies to optimize dairy cattle immunity. Journal of Dairy Science 99: 49674982.

Soriano-Hernández XY, Velasco-Velasco VA, Ruiz-Luna J, Caballero-Gutiérrez PU, Ramírez-Santiago E, Enríquez-del Valle JR (2017) Análisis de Nitrógeno, sólidos suspendidos y disueltos totales en el río Atoyac, Oaxaca. Revista Mexicana de Agroecosistemas 4: 152-163.

Steffan PE, Fiel CA, Ferreyra DA (2012) Endoparasitosis más frecuentes de los rumiantes en sistemas pastoriles de producción. Ed. Tandil Grupo Reencuentro. Argentina. 112p.

Vázquez PVM, Flores CJ, Santiago VC, Herrera RD, Palacios FA, Liébano HE, Pelcastre OA (2004) Frecuencia de nemátodos gastroentéricos en bovinos de tres áreas de clima subtropical húmedo de México. Técnica Pecuaria en México 42: 237-245.

Zajac AM, Conboy GA (2011) Veterinary clinical parasitology. Octava edición. Wiley-Blackwell. lowa, USA. 368p. 